\title{
Versatile multipartite Einstein-Podolsky-Rosen steering via a quantum frequency comb
}

\author{
Yin Cai $\odot,{ }^{1,2}$ Yu Xiang $\odot,{ }^{3,4,5}$ Yang Liu $\odot,{ }^{1,6,7}$ Qiongyi He $\odot,{ }^{3,4,5, *}$ and Nicolas Treps ${ }^{2}$ \\ ${ }^{1}$ Key Laboratory for Physical Electronics and Devices of the Ministry of Education \& Shaanxi Key Lab of Information Photonic Technique, \\ Xi'an Jiaotong University, Xi'an 710049, China \\ ${ }^{2}$ Laboratoire Kastler Brossel, Sorbonne Université, ENS-PSL Research University, Collège de France, CNRS, 4 place Jussieu, \\ F-75252 Paris, France \\ ${ }^{3}$ State Key Laboratory for Mesoscopic Physics, School of Physics, Frontiers Science Center for Nano-optoelectronics, \& Collaborative \\ Innovation Center of Quantum Matter, Peking University, Beijing 100871, China \\ ${ }^{4}$ Beijing Academy of Quantum Information Sciences, Haidian District, Beijing 100193, China \\ ${ }^{5}$ Collaborative Innovation Center of Extreme Optics, Shanxi University, Taiyuan, Shanxi 030006, China \\ ${ }^{6}$ State Key Laboratory of Transient Optics and Photonics, Xi' an Institute of Optics and Precision Mechanics, \\ Chinese Academy of Sciences, Xi'an 710119, China \\ ${ }^{7}$ University of Chinese Academy of Sciences (UCAS), Beijing 100049, China
}

(Received 29 October 2019; revised 18 April 2020; accepted 2 August 2020; published 21 August 2020)

\begin{abstract}
Multipartite Einstein-Podolsky-Rosen steering is an essential resource for quantum communication networks where the reliability of the equipment at all of the nodes cannot be fully trusted. Here, we present the experimental generation of a highly versatile and flexible repository of multipartite steering using an optical frequency comb and ultrafast pulse shaping. Simply modulating the optical spectral resolution of the detection system using the pulse shaper, this scheme is able to produce on-demand four-, eight-, and 16-mode Gaussian steering without changing the photonics architecture. We find that the steerability increases with a higher spectral resolution. For the 16-mode state, we identify as many as 65534 possible steering bipartitions existing in this intrinsic multimode quantum resource, and demonstrate that the prepared state steerability is robust to mode losses. Moreover, we verify four types of monogamy relations of Gaussian steering and demonstrate the constraint imposed by one of them to be strongly lifted. Our method offers a powerful foundation for constructing quantum networks in real-world scenarios.
\end{abstract}

DOI: 10.1103/PhysRevResearch.2.032046

In 1935, Einstein, Podolsky, and Rosen (EPR) [1] pointed out one of the most counterintuitive features of quantum mechanics: The local measurements performed on one system $A$ seem to immediately affect the state of another distant system $B$. The nonlocal effect embodied in this EPR paradox was named "steering" by Schrödinger [2]. In 2007, the concept of steering was rigorously defined $[3,4]$ in view of the local hidden state model, and it was specified that steering allows for the verification of entanglement shared between two remote parties even if one of them is untrusted [5-7]. Different from Bell nonlocality [8] and entanglement [9] where the roles of the involved parties are symmetric, steering is a directional form of nonlocality for which the losses or noises can act asymmetrically [10-12]. For two-party communication, steering has been applied to realize secure quantum teleportation [13-15], one-sided device-independent (1sDI) quantum cryptography [16-19], and subchannel discrimination [20-22].

\footnotetext{
*qiongyihe@pku.edu.cn

Published by the American Physical Society under the terms of the Creative Commons Attribution 4.0 International license. Further distribution of this work must maintain attribution to the author(s) and the published article's title, journal citation, and DOI.
}

When one aims at realistic applications in quantum communication networks where the reliability of devices or the possibility of dishonest measurements are critical, multimode EPR steering is relevant [23-25]. For instance, if for a certain quantum task a given form of multipartite entanglement is required, multipartite steering allows one to verify that this entanglement is present without the need for the full trust of all measurement devices [26]. Recently, the experimental generation of multipartite steering in the continuous-variable (CV) regime has developed rapidly. Armstrong et al. [25] and Deng et al. [27] produced multipartite steering by mixing squeezed light via beam-splitter networks. These experiments, however, require remodifying optical setups for creating different steerable states, and in particular need more squeezed light sources and beam splitters to improve the steerability and enlarge the network, thus lacking versatility and flexibility. In contrast, via parametric down-conversion of a train of ultrafast pulses, multiple frequencies can be quantum correlated simultaneously to generate multimode entanglement from a single cavity [28,29], which allows on-demand generation of multipartite entangled states without changing the optical circuit. This technique offers a convenient and powerful platform for realizing many squeezed spectral/temporal modes copropagating within a single beam [30]. It produces full multipartite entanglement of up to 115974 possible nontrivial mode 
partitions for a ten-mode system [31,32], as well as creates ondemand cluster networks [33]. In particular, these entangled frequency modes can be spatially separated or recombined in free space or fibers within the current optical technologies, i.e., wavelength division multiplexing and demultiplexing in optical telecommunication, and hence, to be further detected and manipulated for various quantum tasks.

Here, we demonstrate multipartite steering between partitions of such an intrinsically multimode resource generated from the parametric down-conversion of an optical frequency comb. The many different steering configurations are obtained without changing the optical setup but simply projecting the state on the required spectral modes, either with pulse-shaped homodyne detection or postprocessing the data from the fully spectrally resolved measurement. We find that the steerability increases when one increases the spectral resolution of the measurement, decomposing the signal spectrum into four-, eight-, and 16-mode states. We also show up to 65534 steering bipartitions coexisting in the multimode quantum system and demonstrate their robustness to the loss of modes. Moreover, we examine the monogamy relations of Gaussian steering, which qualitatively defines the information security among different parties, and show one of the monogamy constraints to be strongly lifted when the detection spectral resolution increases. Our results indicate that this multimode quantum platform is a scalable and versatile resource for multipartite steering. Furthermore, as the relevant modes are the frequency modes, easily manipulated and distributed, the quantum comb is shown to be a very promising candidate to construct quantum communication networks in the real world.

Experimental realization. As illustrated in Fig. 1, a femtosecond optical frequency comb, composed of around $10^{5}$ frequencies centered at $795 \mathrm{~nm}$ and with a $76 \mathrm{MHz}$ spacing, is frequency doubled and pumps a synchronously pumped optical parametric oscillator (SPOPO) below threshold. Within the nonlinear crystal, pairs of photons are generated whose frequency sum matches one of the pump frequency, due to energy conservation. Furthermore, a synchronous cavity ensures that the output field is also a coherent optical frequency comb, matching the source laser spectral properties. The result of this down-conversion process thus forms a multimode quantum frequency comb, with a highly rich correlation pattern in the frequency domain, demonstrated to be fully multipartite [31]. This same state can also be described as a coherent superposition of many copropagating, independently squeezed spectral/temporal modes. This quantum state is interrogated with homodyne detection, in which ultrafast pulse shaping is employed to select the spectral amplitude of the local oscillator (LO), and thus the measured spectral/temporal mode [30].

The bottom plot in Fig. 1 shows how the spectrum of the LO is partitioned: Sixteen spectral bands of equal frequency width are used. Scanning the relative phase between the quantum frequency comb and the LO, the amplitude $\hat{x}$ and phase $\hat{p}$ quadrature of the mode selected by the LO are measured. Shaping the LO into pairs of spectral bands, the correlations are also measured. Note that in our case the cross correlations of the forms $\langle\hat{x} \hat{p}\rangle$ or $\langle\hat{p} \hat{x}\rangle$ are measured to be negligible. From these measurements, the full covariance matrix can be revealed, as was demonstrated in Ref. [30]. In the present

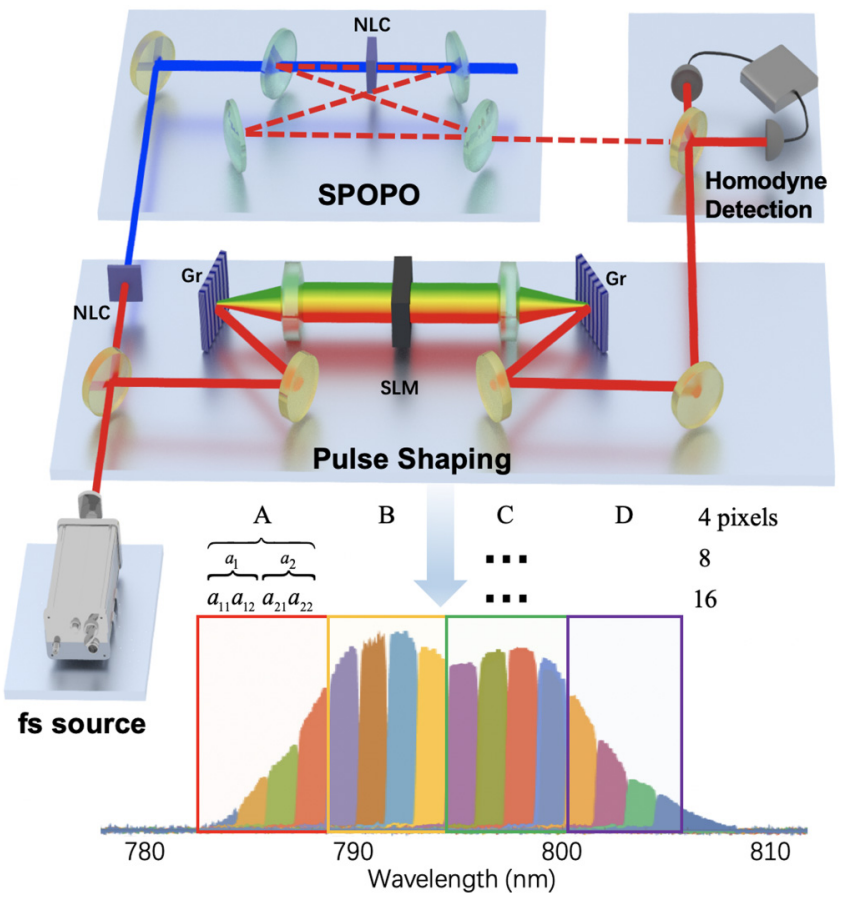

FIG. 1. Experimental setup of the multimode quantum resource via synchronously pumping an optical parametric oscillator (SPOPO). Homodyne detection with pulse shaping on the local oscillator (LO) is applied. The bottom shows the pulse shaping on the LO with a controllable spectral resolution. The spectrum of the LO is divided into four, eight, and 16 spectral bands, respectively. NLC represents a nonlinear crystal, Gr is a grating, and SLM means a spatial light modulator.

case, one can show that the eigenmodes of this covariance matrix display squeezing up to $\sim-5 \mathrm{~dB}$ (see details in the Supplemental Material [34]).

Covariance matrix $(C M)$ and steerability. As explained above, the covariance matrix is originally measured in a basis of 16 equally spaced spectral band modes. Defining $\hat{\vec{\zeta}}=$ $\left(\hat{x}_{1}, \hat{p}_{1}, \ldots, \hat{x}_{i}, \hat{p}_{i}, \ldots\right)$ where $\hat{x}_{i}$ and $\hat{p}_{i}$ are the quadrature operators of the $i$ th spectral band, the covariance matrix elements are formally defined as $\frac{1}{2}\left\langle\hat{\zeta}_{i} \hat{\zeta}_{j}+\hat{\zeta}_{j} \hat{\zeta}_{i}\right\rangle-\left\langle\hat{\zeta}_{i}\right\rangle\left\langle\hat{\zeta}_{j}\right\rangle$. From this matrix, it is possible to derive the covariance matrix of any bipartition, where each party can be composed of several modes. Let us consider that the first party is composed of $m$ modes and the second of $n$ modes, so the covariance matrix can be recast in the form $\sigma_{m n}=\left(\begin{array}{cc}\mathcal{M} & C \\ C^{\top} & { }_{N}\end{array}\right)$ where submatrices $\mathcal{M}, \mathcal{N}$ correspond to the reduced states of each party, respectively, and submatrix $C$ represents the correlation between them.

In practice, we reconstruct the CMs with four, eight, and 16 modes, as plotted on the bottom of Fig. 1. For instance, the spectral component labeled with $A$ is further divided into $\left\{a_{1}, a_{2}\right\}$, and then $\left\{a_{11}, a_{12}, a_{21}, a_{22}\right\}$, and the same operation is performed for the spectral bands $B, C$, and $D$ [34]. The CMs of four-, eight-, and 16-mode states correspond to projecting the same state of the quantum frequency comb onto the spectral band basis with increasing measurement resolution in the pulse-shaping process, without changing any setting of the original resource. The steerability from party $m$ to party $n$ 


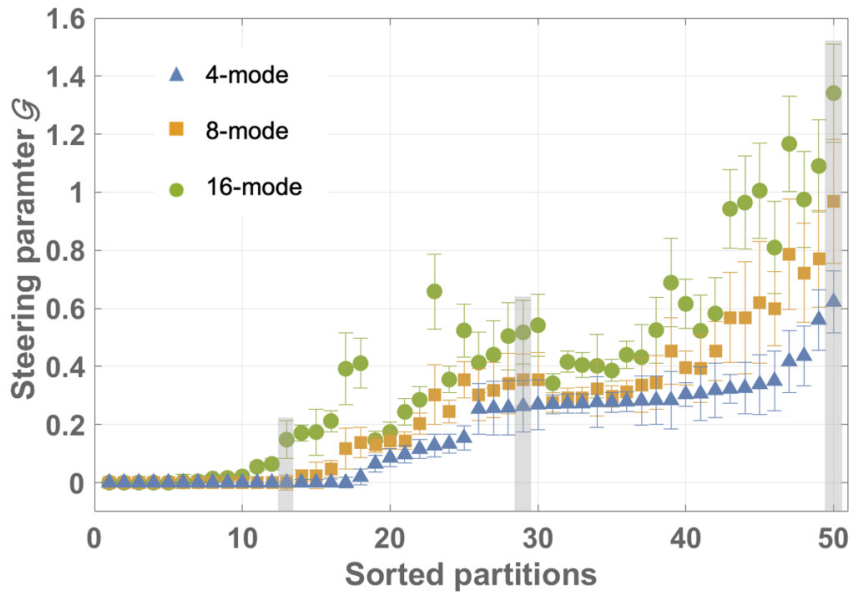

FIG. 2. Multipartite EPR steering among four spectral bands $A B C D$ is measured experimentally with 4 (blue triangles), 8 (orange squares), and 16 (green dots) spectral pixels, respectively. The partitions are arranged from small to large according to the steerability of the four-mode state. Marks on the same vertical line correspond to the steering between the same spectral bands with different spectral pixels. The error bars arise from the uncertainty of quadrature correlation measurements via homodyne detection. These results are close to those calculated with the ideal quantum source [34].

is quantified by [24]

$$
\mathcal{G}^{m \rightarrow n}\left(\sigma_{m n}\right)=\max \left\{0,-\sum_{i: \bar{v}_{i}^{m n / m}<1} \ln \left(\bar{v}_{i}^{m n / m}\right)\right\},
$$

where $\left\{\bar{v}_{i}^{m n / m}\right\}$ are the symplectic eigenvalues of $\bar{\sigma}_{m n / m}=$ $\mathcal{N}-C^{\top} \mathcal{M}^{-1} \mathcal{C}$, which is the Schur complement of partition $m$. Under Gaussian local operations and classical communication, this quantity $\mathcal{G}^{m \rightarrow n}$ is always monotone. A zero $\mathcal{G}^{m \rightarrow n}$ means that $m$ is not able to steer $n$ by Gaussian measurements, but it may succeed with non-Gaussian measurements [39].

Results. Figure 2 shows multipartite steering among the four spectral bands $A B C D$ when the whole spectrum is divided into 4 (blue triangles), 8 (orange squares), or 16 (green dots) pixels. Three marks on the same vertical line correspond to the bipartition steerability between identical spectral bands but measured with different resolutions. Note that here we show all possible nontrivial partitions for the four-mode state, while for comparison we only show the partitions of eightand 16-mode states that are correspondingly subdivided from the four spectral bands $A B C D$ without taking into account the mixtures among them. Three representative groups (gray slices) are delineated in Table I. Interestingly, although the spectral components are fixed, the steerability rises sharply with the increase of measurement resolutions for all possible partitions. For instance, $\mathcal{G}^{(A B) \rightarrow C}<\mathcal{G}^{\left(a_{1}, a_{2}, b_{1}, b_{2}\right) \rightarrow\left(c_{1}, c_{2}\right)}<$ $\mathcal{G}^{\left(a_{11}, a_{12}, a_{21}, a_{22}, b_{11}, b_{12}, b_{21}, b_{22}\right) \rightarrow\left(c_{11}, c_{12}, c_{21}, c_{22}\right)}$. This phenomenon can have several origins. First, by increasing the resolution, one better resolves the spectral shape of the eigenmodes, hence the measured squeezing increases and thus the steerability. Furthermore, adding more parties increases the number of extracted modes, the parameter which also influences the steerability as we will study in the last section
TABLE I. Steerability with different partitions (one-to-multi, multi-to-one and multi-to-multi) and their respective spectral resolutions.

\begin{tabular}{lcr}
\hline \hline Resolution & Partitions & \multicolumn{1}{c}{$\mathcal{G}$} \\
\hline 4 & $\mathcal{G}^{A \rightarrow(B C)}$ & $0 \pm 0.0043$ \\
8 & $\mathcal{G}^{\left(a_{1}, a_{2}\right) \rightarrow\left(b_{1}, b_{2}, c_{1}, c_{2}\right)}$ & $0 \pm 0.0270$ \\
16 & $\mathcal{G}^{\left(a_{11}, a_{12}, a_{21}, a_{22}\right) \rightarrow\left(b_{11}, b_{12}, \ldots, c_{21}, c_{22}\right)}$ & $0.1481 \pm 0.0714$ \\
4 & $\mathcal{G}^{(A B) \rightarrow C}$ & $0.2633 \pm 0.0881$ \\
8 & $\mathcal{G}^{\left(a_{1}, a_{2}, b_{1}, b_{2}\right) \rightarrow\left(c_{1}, c_{2}\right)}$ & $0.3537 \pm 0.0927$ \\
16 & $\mathcal{G}^{\left(a_{11}, a_{12}, \ldots, b_{21}, b_{22}\right) \rightarrow\left(c_{11}, c_{12}, c_{21}, c_{22}\right)}$ & $0.5177 \pm 0.1139$ \\
4 & $\mathcal{G}^{(C D) \rightarrow(A B)}$ & $0.6226 \pm 0.0963$ \\
8 & $\mathcal{G}^{\left(c_{1}, c_{2}, d_{1}, d_{2}\right) \rightarrow\left(a_{1}, a_{2}, b_{1}, b_{2}\right)}$ & $0.9692 \pm 0.1372$ \\
16 & $\mathcal{G}^{\left(c_{11}, c_{12}, \ldots, d_{21}, d_{22}\right) \rightarrow\left(a_{11}, a_{12}, \ldots, b_{21}, b_{22}\right)}$ & $1.3415 \pm 0.1836$ \\
\hline \hline
\end{tabular}

of this Rapid Communication on monogamy. Hence, we see that our scheme allows for the preparation of versatile multipartite EPR steering and simultaneously increasing the steerability simply by modulating the spectral resolution of the detection system, i.e., acting on the spectral shape of the LO. Moreover, we can generate richer steering structures by asymmetrically adjusting the pulse shaping. For example, by increasing the resolutions either on the steering party or steered party, one-way steering is realized [34].

Owing to the high spectral resolution of our pulse shaper, EPR steering is finally certified for 65534 possible bipartitions for the 16-mode system. Figure 3(a) illustrates the steerability $\mathcal{G}^{m \rightarrow n}$ between the $m \mid n$ bipartition $(m+n=16)$. As the prepared state is not a pure state, the trend of steerability is not ideally symmetric, nevertheless the highest steerability still occurs when the steering and steered party are symmetric and cover the full spectrum, i.e., $\mathcal{G}^{8 \rightarrow 8} \approx 1.34$ shown in the last line of Table I. The amount of Gaussian steering directly bounds the secure key rate in the 1sDI quantum cryptography, i.e., $\mathcal{G}>\ln (e / 2)$, to obtain a nonzero key rate $[19,24]$, which is satisfied by most of data shown in Fig. 3(a).

We also analyze the robustness of multipartite steering against the loss of one or several modes during the transmission, as shown in Fig. 3(b). We remove up to six modes one by one, i.e., removing $\left\{a_{11}\right\},\left\{a_{11}, a_{12}\right\},\left\{a_{11}, a_{12}, a_{21}\right\}, \ldots$, $\left\{a_{11}, a_{12}, a_{21}, a_{22}, b_{11}, b_{12}\right\}$ from the system, then figure out multipartite steering for all possible partitions of the remaining modes $\mathcal{G}^{m \rightarrow n}(m+n=15,14, \ldots, 10)$. As the information is multimode and spreads across the full quantum comb, the system keeps its steerable capability with 32765,16379 , 8185, 4083, 2025, and 943 possible nontrivial bipartitions for each reduced systems, respectively. Hence, a fertile and modeloss resistant repository of the quantum correlations such as multipartite steering and entanglement [31] is analyzed systemically.

Finally, to characterize more thoroughly the multimode aspect of the achieved resources, we investigate an important feature called monogamy relations, which reveal how EPR steering can be distributed over many different parties. Recently, various kinds of monogamy relations have been studied theoretically [40-46] and demonstrated experimentally $[27,47]$ both for continuous and discrete variable systems. Within the accurate quantification of bipartite Gaussian 

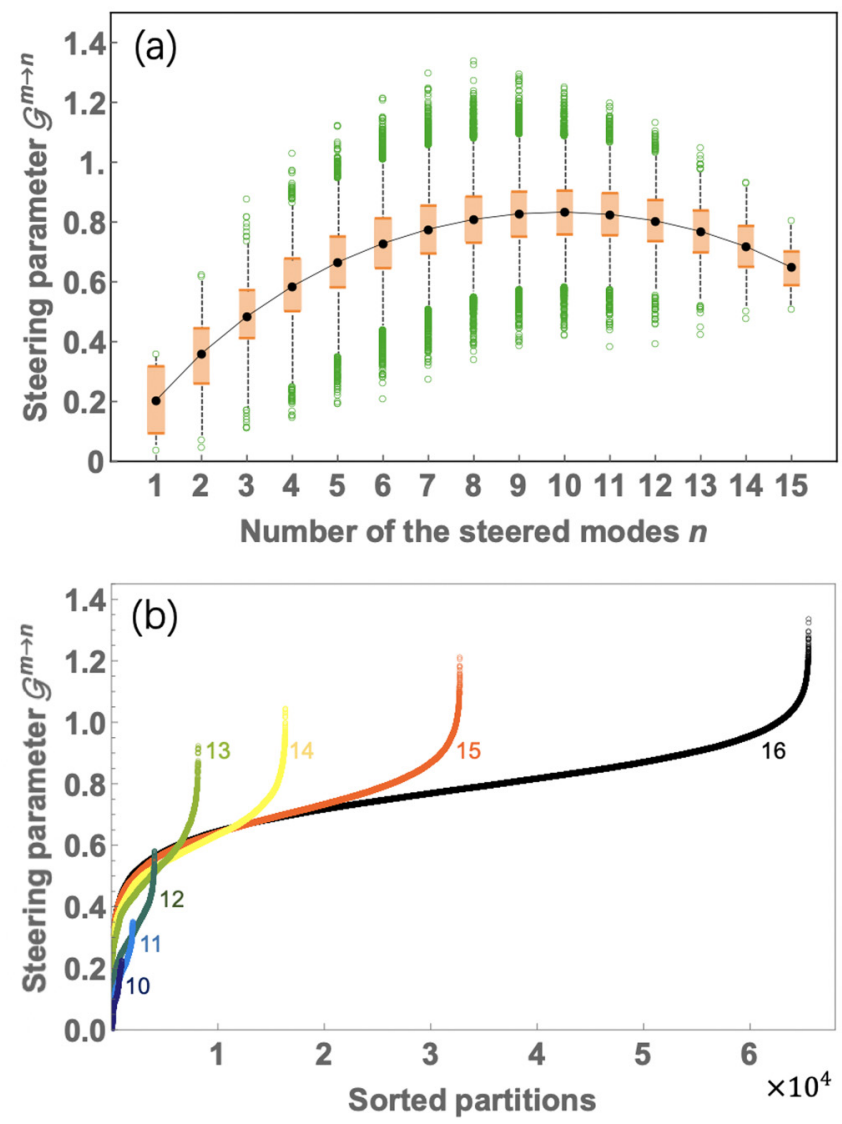

FIG. 3. Multipartite steering among 16 spectral band modes. (a) represents the steerability $\mathcal{G}^{m \rightarrow n}(m+n=16)$ for all possible 65534 partitions. The black dots are the average of steerability for each partition. $50 \%$ of data display around the center represented by the orange boxes, and nearly $97 \%$ of data are covered between the whiskers. (b) shows EPR steering against the mode loss of $\left\{a_{11}\right\},\left\{a_{11}, a_{12}\right\},\left\{a_{11}, a_{12}, a_{21}\right\}, \ldots,\left\{a_{11}, a_{12}, a_{21}, \ldots, b_{11}, b_{12}\right\}$, respectively.

steering $\mathcal{G}^{m \rightarrow n}$, we show that four types of monogamy constraints [40-44] are validated and one of them is strongly lifted in some cases.

As shown in Table II, the type-I monogamy relation implies that two distinct modes cannot steer a third mode simultaneously by Gaussian measurements, i.e., Gaussian steering is exclusive [41]. The type-II monogamy relation is a generalization of type I where two steering parties comprise an arbitrary number of modes [42,43]. Then, the Coffman-Kundu-Wootters (CKW)-type monogamy relation was proposed showing that the sum of steerability between any two modes cannot exceed their intergroup steerability [40], which reads $\mathcal{G}^{\left(i_{1}, \ldots, i_{m}\right) \rightarrow j} \geqslant \mathcal{G}^{i_{1} \rightarrow j}+\cdots+\mathcal{G}^{i_{m} \rightarrow j}$ and $\mathcal{G}^{i \rightarrow\left(j_{1}, \ldots, j_{n}\right)} \geqslant \mathcal{G}^{i \rightarrow j_{1}}+\cdots+\mathcal{G}^{i \rightarrow j_{n}} \quad(n, m \geqslant 2)$. It was recently extended to a general case (type IV) where both the steering party and steered party contain more than one mode [44], which takes the form $\mathcal{G}^{\left(i_{1}, \ldots, i_{m}\right) \rightarrow\left(j_{1}, \ldots, j_{n}\right)} \geqslant \mathcal{G}^{\left(i_{1}, \ldots, i_{m}\right) \rightarrow j_{1}}+\cdots+\mathcal{G}^{\left(i_{1}, \ldots, i_{m}\right) \rightarrow j_{n}} \quad$ and $\mathcal{G}^{\left(i_{1}, \ldots, i_{m}\right) \rightarrow\left(j_{1}, \ldots, j_{n}\right)} \geqslant \mathcal{G}^{i_{1} \rightarrow\left(j_{1}, \ldots, j_{n}\right)}+\cdots+\mathcal{G}^{i_{m} \rightarrow\left(j_{1}, \ldots, j_{n}\right)} \quad$ (the latter one can be lifted in some cases).
TABLE II. Depiction of four types of monogamy relations and their corresponding experimental cases.

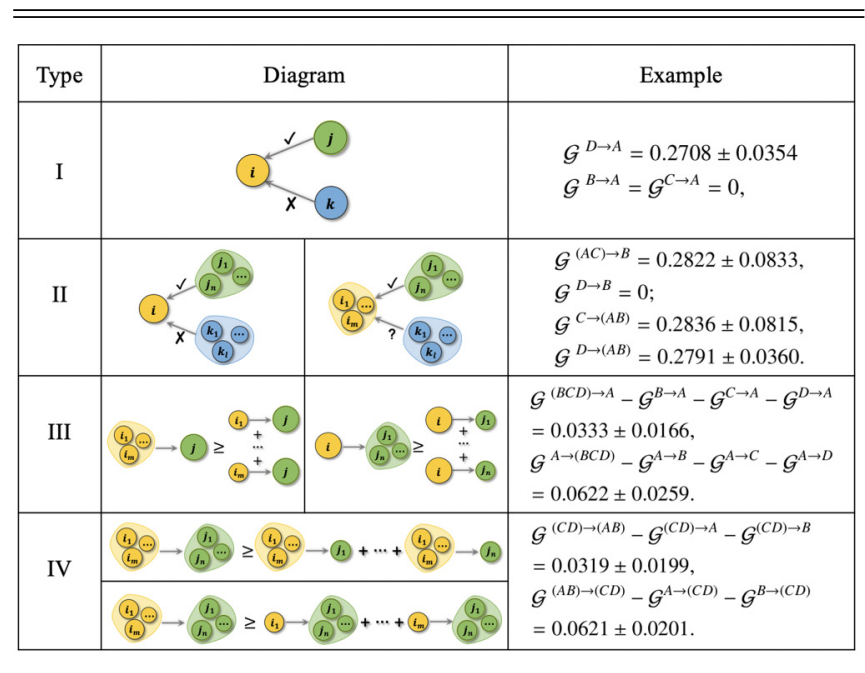

Our experimental results demonstrate that type I, CKW type, and type IV remain valid for all possible nontrivial mode partitions regardless of the spectral resolutions. Some of the results are shown in Table II. But the type-II monogamy constraint can be lifted when the steered party is made of more than one mode, as was predicted theoretically [43]. Here, we present an experimental observation that $C \rightarrow(A B)$ and $D \rightarrow(A B)$ are steerable simultaneously by Gaussian measurements, with $\mathcal{G}^{C \rightarrow(A B)}=0.2836 \pm$ 0.0815 and $\mathcal{G}^{D \rightarrow(A B)}=0.2791 \pm 0.0360$ even if the spectrum is divided into only 4 pixels. When the detection spectral resolution increases, the type-II monogamy constraint will be further lifted with more refined spectral band modes, e.g., $\mathcal{G}^{\left(c_{11}, c_{12}, c_{21}, c_{22}\right) \rightarrow\left(a_{11}, a_{12}, \ldots, b_{21}, b_{22}\right)}=0.5253 \pm 0.1117$ and $\mathcal{G}^{\left(d_{11}, d_{12}, d_{21}, d_{22}\right) \rightarrow\left(a_{11}, a_{12}, \ldots, b_{21}, b_{22}\right)}=0.4405 \pm 0.0520$ with 16 pixels. The error bars in Fig. 2 and the tables arise from the uncertainty of quadrature correlation measurements via homodyne detection. We drew Gaussian-distributed random values with standard deviations (s.d.'s) matching those of the experimentally measured quadrature squeezing values [33]. Using these random numbers, numerical steerabilities are obtained by simulating with the use of Eq. (1).

Conclusion. In summary, we demonstrate a versatile and flexible repository of multipartite EPR steering within an optical frequency comb and ultrafast pulse shaping. Owing to this intrinsic multimode resource, we are able to prepare on-demand Gaussian steerable states with four, eight, and 16 modes through a variable detection spectral resolution without changing the photonics architecture. Increasing the spectral resolution, more independent squeezed modes as well as better squeezing can be extracted and thus the steerability between the according bipartition also increases sharply. Then, we identify multipartite steering in all of the 65534 nontrivial bipartitions of the 16-mode state. As the quantum comb carries all information, the prepared 16-mode state can maintain high steerability in the presence of mode loss. Finally, we verify four types of monogamy relations of Gaussian steering and demonstrate the type-II monogamy constraint can be strongly lifted. 
It is theoretically predicted that this quantum comb source can lead to up to 100 independent squeezed modes given that one can enhance the energy and spectral bandwidth of the LO light and the spectral resolutions [48], which provides a scalable and versatile platform for multipartite steering. Although the current experimental implementation is not yet a practical application to quantum secure communications, where the modes from the frequency comb need to be spatially separated and distributed to spatially distant parties, however, a standard technique in telecoms or spectroscopy can be used for separating frequency bins via dispersive optics. For instance, we adapted multimode homodyne detection, which has been performed in the spatial domain [49], to the frequency domain and used it to simultaneously measure the prepared modes within frequency pixels [50-52]. We have demonstrated that steering remains in that situation. The maturity of these techniques, at the heart of modern optical telecommunications, allows for low-loss operation (see more details in the Supplemental Material [34]). Such a property contributes to make the quantum comb a powerful candidate to construct quantum communication networks in the real world.

Beyond the largest impacts of EPR steering made in its myriad 1sDI scenarios [53], interestingly, a recent study shows that EPR steering is directly connected to the ability to transfer Wigner negativity [54], which is identified as an essential resource to reach a quantum computational advantage with CV systems [55]. Yet, the study of non-Gaussian steering is facing many challenges and existing numerous open questions, e.g., a complete characterization and quantification of quantum steering and the subchannel discrimination task in the non-Gaussian scenario, which is even harder to implement in a multimode scenario. Therefore, the present multipartite steering Gaussian states together with a controllable nonGaussian operation such as photon subtraction [56] are useful for studying such applications in CV quantum optics.

Acknowledgments. We acknowledge insightful comments and discussions on multimode entanglement and experiments with C. Fabre and J. Roslund. This work is supported by the National Key R\&D Program of China (Grants No. 2017YFA0303700, No. 2018YFB1107205, and No. 2016YFA0301302), the National Natural Science Foundation of China (Grants No. 61975159, No. 11904279, No. 11622428, No. 61675007, and No. 11975026), the National Science Foundation of Jiangsu Province (Grant No. BK20180322), and the French National Research Agency projects COMB and SPOCQ. Y.X. recognizes support from the National Postdoctoral Program for Innovative Talents (BX20180015), and China Postdoctoral Science Foundation (2019M650291). Q.H. is also thankful for partial support from Beijing Natural Science Foundation (Grant No. Z190005) and the Key R\&D Program of Guangdong Province (Grant No. 2018B030329001). N.T. acknowledges the financial support from the Institut Universitaire de France.

Y.C., Y.X., and Y.L. contributed equally to this work.
[1] A. Einstein, B. Podolsky, and N. Rosen, Can quantummechanical description of physical reality be considered complete, Phys. Rev. 47, 777 (1935).

[2] E. Schrödinger, Discussion of probability relations between separated systems, Proc. Cambridge Philos. Soc. 31, 555 (1935).

[3] H. M. Wiseman, S. J. Jones, and A. C. Doherty, Steering, Entanglement, Nonlocality, and the Einstein-Podolsky-Rosen Paradox, Phys. Rev. Lett. 98, 140402 (2007).

[4] S. J. Jones, H. M. Wiseman, and A. C. Doherty, Entanglement, Einstein-Podolsky-Rosen correlations, Bell nonlocality, and steering, Phys. Rev. A 76, 052116 (2007).

[5] E. G. Cavalcanti, M. J. W. Hall, and H. M. Wiseman, Entanglement verification and steering when Alice and Bob cannot be trusted, Phys. Rev. A 87, 032306 (2013).

[6] M. D. Reid, P. D. Drummond, W. P. Bowen, E. G. Cavalcanti, P. K. Lam, H. A. Bachor, U. L. Andersen, and G. Leuchs, Colloquium: The Einstein-Podolsky-Rosen paradox: From concepts to applications, Rev. Mod. Phys. 81, 1727 (2009).

[7] R. Uola, A. C. S. Costa, H. Chau Nguyen, and O. Gühne, Quantum steering, Rev. Mod. Phys. 92, 015001 (2020).

[8] N. Brunner, D. Cavalcanti, S. Pironio, V. Scarani, and S. Wehner, Bell nonlocality, Rev. Mod. Phys. 86, 419 (2014).

[9] R. Horodecki, P. Horodecki, M. Horodecki, and K. Horodecki, Quantum entanglement, Rev. Mod. Phys. 81, 865 (2009).

[10] S. L. W. Midgley, A. J. Ferris, and M. K. Olsen, Asymmetric Gaussian steering: When Alice and Bob disagree, Phys. Rev. A 81, 022101 (2010); S. P. Walborn, A. Salles, R. M. Gomes, F. Toscano, and P. H. Souto Ribeiro, Revealing Hidden
Einstein-Podolsky-Rosen Nonlocality, Phys. Rev. Lett. 106, 130402 (2011); J. Schneeloch, C. J. Broadbent, S. P. Walborn, E. G. Cavalcanti, and J. C. Howell, Einstein-Podolsky-Rosen steering inequalities from entropic uncertainty relations, Phys. Rev. A 87, 062103 (2013); J. Bowles, T. Vertesi, M. T. Quintino, and N. Brunner, One-Way Einstein-Podolsky-Rosen Steering, Phys. Rev. Lett. 112, 200402 (2014); B. Opanchuk, L. Arnaud, and M. D. Reid, Detecting faked continuous-variable entanglement using one-sided device-independent entanglement witnesses, Phys. Rev. A 89, 062101 (2014).

[11] Q. Y. He, Q. H. Gong, and M. D. Reid, Classifying Directional Gaussian Entanglement, Einstein-Podolsky-Rosen Steering, and Discord, Phys. Rev. Lett. 114, 060402 (2015).

[12] L. Rosales-Zárate, R. Y. Teh, S. Kiesewetter, A. Brolis, K. $\mathrm{Ng}$, and M. D. Reid, Decoherence of Einstein-Podolsky-Rosen steering, J. Opt. Soc. Am. B 32, A82 (2015).

[13] M. D. Reid, Signifying quantum benchmarks for qubit teleportation and secure quantum communication using EinsteinPodolsky-Rosen steering inequalities, Phys. Rev. A 88, 062338 (2013).

[14] Q. He, L. Rosales-Zárate, G. Adesso, and M. D. Reid, Secure Continuous Variable Teleportation and Einstein-PodolskyRosen Steering, Phys. Rev. Lett. 115, 180502 (2015).

[15] C.-Y. Chiu, N. Lambert, T.-L. Liao, F. Nori, and C.-M. Li, Nocloning of quantum steering, npj Quantum Inf. 2, 16020 (2016).

[16] M. Tomamichel and R. Renner, Uncertainty Relation for Smooth Entropies, Phys. Rev. Lett. 106, 110506 (2011).

[17] C. Branciard, E. G. Cavalcanti, S. P. Walborn, V. Scarani, and H. M. Wiseman, One-sided device-independent quantum 
key distribution: Security, feasibility, and the connection with steering, Phys. Rev. A 85, 010301 (2012).

[18] T. Gehring, V. Händchen, J. Duhme, F. Furrer, T. Franz, C. Pacher, R. F. Werner, and R. Schnabel, Implementation of continuous-variable quantum key distribution with composable and one-sided-device-independent security against coherent attacks, Nat. Commun. 6, 8795 (2015).

[19] N. Walk, S. Hosseini, J. Geng, O. Thearle, J. Y. Haw, S. Armstrong, S. M. Assad, J. Janoušek, T. C. Ralph, T. Symul, H. M. Wiseman, and P. K. Lam, Experimental demonstration of Gaussian protocols for one-sided device-independent quantum key distribution, Optica 3, 634 (2016).

[20] M. Piani and J. Watrous, Necessary and Sufficient Quantum information Characterization of Einstein-Podolsky-Rosen Steering, Phys. Rev. Lett. 114, 060404 (2015).

[21] S.-L. Chen, C. Budroni, Y.-C. Liang, and Y.-N. Chen, Natural Framework for Device-Independent Quantification of Quantum Steerability, Measurement Incompatibility, and Self-Testing, Phys. Rev. Lett. 116, 240401 (2016).

[22] K. Sun, X.-J. Ye, Y. Xiao, X.-Y. Xu, Y.-C. Wu, J.-S. Xu, J.-L. Chen, C.-F. Li, and G.-C. Guo, Demonstration of EinsteinPodolsky-Rosen steering with enhanced subchannel discrimination, npj Quantum Inf. 4, 12 (2018).

[23] Q. Y. He and M. D. Reid, Genuine Multipartite EinsteinPodolsky-Rosen Steering, Phys. Rev. Lett. 111, 250403 (2013).

[24] I. Kogias, A. R. Lee, S. Ragy, and G. Adesso, Quantification of Gaussian Quantum Steering, Phys. Rev. Lett. 114, 060403 (2015).

[25] S. Armstrong, M. Wang, R. Y. Teh, Q. H. Gong, Q. Y. He, J. Janousek, H. A. Bachor, M. D. Reid, and P. K. Lam, Multipartite Einstein-Podolsky-Rosen steering and genuine tripartite entanglement with optical networks, Nat. Phys. 11, 167 (2015).

[26] I. Kogias, Y. Xiang, Q. Y. He, and G. Adesso, Unconditional security of entanglement-based continuous-variable quantum secret sharing, Phys. Rev. A 95, 012315 (2017).

[27] X. W. Deng, Y. Xiang, C. Tian, G. Adesso, Q. Y. He, Q. H. Gong, X. L. Su, C. D. Xie, and K. C. Peng, Demonstration of Monogamy Relations for Einstein-Podolsky-Rosen Steering in Gaussian Cluster States, Phys. Rev. Lett. 118, 230501 (2017).

[28] O. Pinel, P. Jian, R. M. de Araújo, J. Feng, B. Chalopin, C. Fabre, and N. Treps, Generation and Characterization of Multimode Quantum Frequency Combs, Phys. Rev. Lett. 108, 083601 (2012).

[29] M. Pysher, Y. Miwa, R. Shahrokhshahi, R. Bloomer, and O. Pfister, Parallel Generation of Quadripartite Cluster Entanglement in the Optical Frequency Comb, Phys. Rev. Lett. 107, 030505 (2011).

[30] J. Roslund, R. M. de Araújo, S. Jiang, C. Fabre, and N. Treps, Wavelength-multiplexed quantum networks with ultrafast frequency combs, Nat. Photonics 8, 109 (2014).

[31] S. Gerke, J. Sperling, W. Vogel, Y. Cai, J. Roslund, N. Treps, and C. Fabre, Full Multipartite Entanglement of Frequency-Comb Gaussian States, Phys. Rev. Lett. 114, 050501 (2015).

[32] S. Gerke, J. Sperling, W. Vogel, Y. Cai, J. Roslund, N. Treps, and C. Fabre, Multipartite Entanglement of a Two-Separable State, Phys. Rev. Lett. 117, 110502 (2016).

[33] Y. Cai, J. Roslund, G. Ferrini, F. Arzani, X. Xu, C. Fabre, and N. Treps, Multimode entanglement in reconfigurable graph states using optical frequency combs, Nat. Commun. 8, 15645 (2017).

[34] See Supplemental Material at http://link.aps.org/supplemental/ 10.1103/PhysRevResearch.2.032046 for details of the squeezing level and CMs of prepared states, comparisons with the results created from pure squeezed input states or a single mode squeezed state, generation of one-way steering via asymmetric control, and separation of the entangled modes, which includes Refs. [35-38].

[35] R. Simon, S. Chaturvedi, and V. Srinivasan, Congruences and canonical forms for a positive matrix: Application to the Schweinler-Wigner extremum principle, J. Math. Phys. 40, 3632 (1999).

[36] S. Wollmann, N. Walk, A. J. Bennet, H. M. Wiseman, and G. J. Pryde, Observation of Genuine One-Way Einstein-PodolskyRosen Steering, Phys. Rev. Lett. 116, 160403 (2016).

[37] K. Sun, X. J. Ye, J. S. Xu, X. Y. Xu, J. S. Tang, Y. C. Wu, J. L. Chen, C. F. Li, and G. C. Guo, Experimental Quantification of Asymmetric Einstein-Podolsky-Rosen Steering, Phys. Rev. Lett. 116, 160404 (2016).

[38] V. Händchen, T. Eberle, S. Steinlechner, A. Samblowski, T. Franz, R. F. Werner, and R. Schnabel, Observation of oneway Einstein-Podolsky-Rosen steering, Nat. Photonics 6, 596 (2012).

[39] Y. Xiang, B. Xu, L. Mišta, Jr., T. Tufarelli, Q. Y. He, and G. Adesso, Investigating Einstein-Podolsky-Rosen steering of continuous-variable bipartite states by non-Gaussian pseudospin measurements, Phys. Rev. A 96, 042326 (2017).

[40] Y. Xiang, I. Kogias, G. Adesso, and Q. Y. He, Multipartite Gaussian steering: Monogamy constraints and quantum cryptography applications, Phys. Rev. A 95, 010101(R) (2017).

[41] M. D. Reid, Monogamy inequalities for the Einstein-PodolskyRosen paradox and quantum steering, Phys. Rev. A 88, 062108 (2013).

[42] S.-W. Ji, M. S. Kim, and H. Nha, Quantum steering of multimode Gaussian states by Gaussian measurements: Monogamy relations and the Peres conjecture, J. Phys. A: Math. Theor. 48, 135301 (2015).

[43] G. Adesso and R. Simon, Strong subadditivity for logdeterminant of covariance matrices and its applications, J. Phys. A: Math. Theor. 49, 34LT02 (2016).

[44] L. Lami, C. Hirche, G. Adesso, and A. Winter, Schur Complement Inequalities for Covariance Materices and Monogamy of Quantum Correlations, Phys. Rev. Lett. 117, 220502 (2016).

[45] B. Regula, A. Osterloh, and G. Adesso, Strong monogamy inequalities for four qubits, Phys. Rev. A 93, 052338 (2016).

[46] S. M. Cheng, A. Milne, M. J. W. Hall, and H. M. Wiseman, Volume monogamy of quantum steering ellipsoids for multiqubit systems, Phys. Rev. A 94, 042105 (2016).

[47] C. Zhang, S. Cheng, L. Li, Q. Liang, B. Liu, Y. Huang, C. Li, G. Guo, M. J. W. Hall, H. M. Wiseman, and G. J. Pryde, Experimental Validation of Quantum Steering Ellipsoids and Tests of Volume Monogamy Relations, Phys. Rev. Lett. 122, 070402 (2019).

[48] G. Patera, N. Treps, C. Fabre, and G. J. de Valcarcel, Quantum theory of synchronously pumped type I optical parametric oscillators: Characterization of the squeezed supermodes, Eur. Phys. J. D 56, 123 (2010). 
[49] S. Armstrong, J.-F. Morizur, J. Janousek, B. Hage, N. Treps, P.-K. Lam, and H-A. Bachor, Programmable multimode quantum networks, Nat. Commun. 3, 1026 (2012).

[50] G. Ferrini, J.-P. Gazeau, T. Coudreau, C. Fabre, and N. Treps, Compact Gaussian quantum computation by multipixel homodyne detection, New J. Phys. 15, 093015 (2013).

[51] S. De, V. Thiel, J. Roslund, C. Fabre, and N. Treps, Modal analysis for noise characterization and propagation in a femtosecond oscillator, Opt. Lett. 44, 3992 (2019).

[52] Y. Cai, J. Roslund, V. Thiel, C. Fabre, and N. Treps, Quantum enhanced measurement of an optical frequency comb, arXiv:2003.05833.
[53] D. Cavalcanti and P. Skrzypczyk, Quantum steering: A review with focus on semidefinite programming, Rep. Prog. Phys. 80, 024001 (2017).

[54] M. Walschaers and N. Treps, Remote Generation of Wigner Negativity through Einstein-Podolsky-Rosen Steering, Phys. Rev. Lett. 124, 150501 (2020).

[55] N. C. Menicucci, P. van Loock, M. Gu, C. Weedbrook, T. C. Ralph, and M. A. Nielsen, Universal Quantum Computation with Continuous-Variable Cluster States, Phys. Rev. Lett. 97, 110501 (2006).

[56] Y.-S. Ra, A. Dufour, M. Walschaers, C. Jacquard, T. Michel, C. Fabre, and N. Treps, Non-Gaussian quantum states of a multimode light field, Nat. Phys. 16, 144 (2020). 